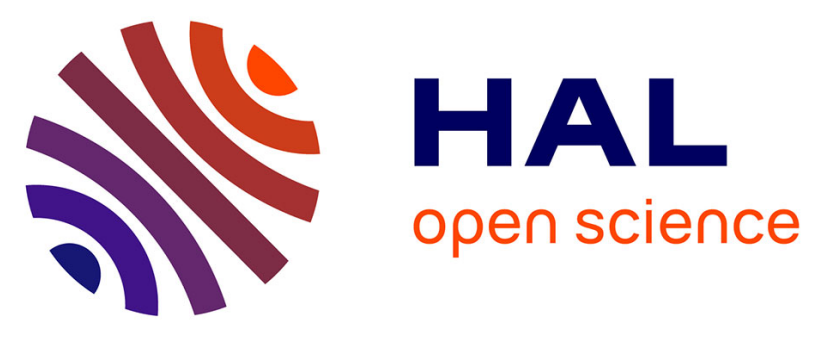

\title{
The Tight Game: Implicit Force Intervention in Inter-personal Physical Interactions on Playing Tug of War
}

\author{
Azumi Maekawa, Shunichi Kasahara, Hiroto Saito, Daisuke Uriu, \\ Gowrishankar Ganesh, Masahiko Inami
}

\section{To cite this version:}

Azumi Maekawa, Shunichi Kasahara, Hiroto Saito, Daisuke Uriu, Gowrishankar Ganesh, et al.. The Tight Game: Implicit Force Intervention in Inter-personal Physical Interactions on Playing Tug of War. SIGGRAPH 2020: Special Interest Group on Computer Graphics and Interactive Techniques Conference, Aug 2020, Virtual, United States. pp.10:1-10:2, 10.1145/3388534.3407301 . hal-03009636

\section{HAL Id: hal-03009636 https://hal.science/hal-03009636}

Submitted on 17 Nov 2020

HAL is a multi-disciplinary open access archive for the deposit and dissemination of scientific research documents, whether they are published or not. The documents may come from teaching and research institutions in France or abroad, or from public or private research centers.
L'archive ouverte pluridisciplinaire HAL, est destinée au dépôt et à la diffusion de documents scientifiques de niveau recherche, publiés ou non, émanant des établissements d'enseignement et de recherche français ou étrangers, des laboratoires publics ou privés. 


\section{The Tight Game: Implicit Force Intervention in Inter-personal Physical Interactions on Playing Tug of War}

\author{
Azumi Maekawa ${ }^{* \dagger}$ \\ azumi@star.rcast.u-tokyo.ac.jp \\ Daisuke Uriu $^{\dagger}$ \\ uriu@star.rcast.u-tokyo.ac.jp
}

\author{
Shunichi Kasahara ${ }^{* \dagger \ddagger}$ \\ kasahara@star.rcast.u-tokyo.ac.jp \\ Ganesh Gowrishankar§ \\ gans_gs@hotmail.com
}

\author{
Hiroto Saito ${ }^{\dagger}$ \\ hiroto.saito@star.rcast.u-tokyo.ac.jp \\ Masahiko Inami ${ }^{\dagger}$ \\ inami@star.rcast.u-tokyo.ac.jp
}
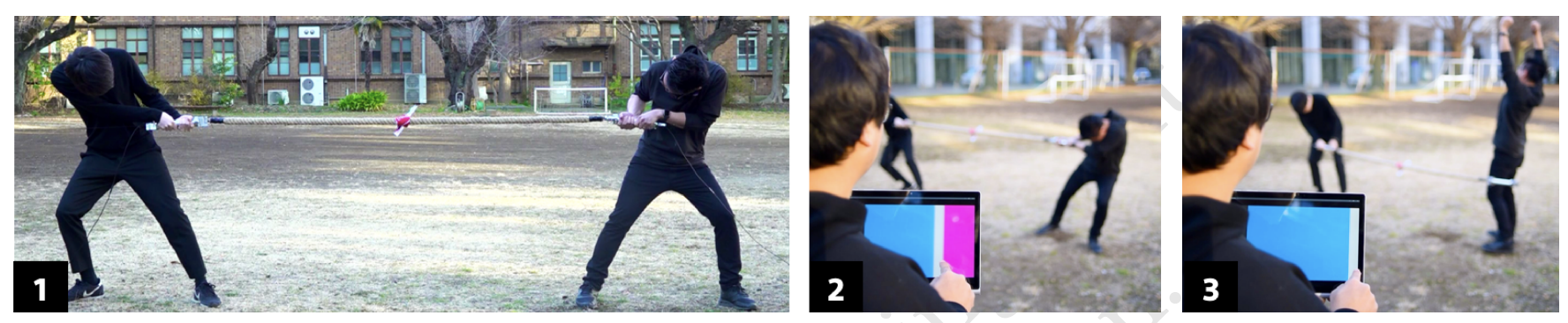

Figure 1: 1) A tug of war fight by two players began. 2) However, another person (as 'mediator') is controlling the game without noticed by the players. 3) The players were totally engaged with the game since they believed to play a tight game.

\begin{abstract}
Physical assistance alleviates individual differences of abilities between players to play well balanced inter-personal physical games. However, the 'explicit' intervention in the games ruins the sense of agency of the players, and loose engagements both of player and audience. We propose an implicit physical intervention system "The Tight Game" for 'Tug of War' a one-dimensional physical game. Our system includes four force sensors in a tug and two hidden high torque motors to provide realtime physical assistance. We designed the implicit physical assistance by leveraging human recognition of the external force in physical actions. In The Tight Game, one pair of players pulls the tug each other, and they believe that they are playing a well balanced tight game. But the system or even another person enables to mediate the game while the players will not recognize the physical interventions.
\end{abstract}

\section{CCS CONCEPTS}

- Human-centered computing $\rightarrow$ Haptic devices; Gestural input.

*Both authors contributed equally to this research.

${ }^{\dagger}$ Research Center for Advanced Science and Technology, The University of Tokyo

¥Sony Computer Science Laboratories

${ }^{\S}$ Centre National de la Recherche Scientifique

\section{Unpublished working draft. Not for distribution.}

\section{KEYWORDS}

Sense of Agency; Force Intervention; Inter-personal Interaction

\section{ACM Reference Format:}

Azumi Maekawa, Shunichi Kasahara, Hiroto Saito, Daisuke Uriu, Ganesh Gowrishankar, and Masahiko Inami. 2020. The Tight Game: Implicit Force Intervention in Inter-personal Physical Interactions on Playing Tug of War. In Proceedings of SIGGRAPH '20 Emerging Technologies, fuly 19-23, 2020, Washington, D.C.. ACM, New York, NY, USA, 2 pages. https://doi.org/10. $1145 / 1234567.9999999$

\section{INTRODUCTION}

Tight games, especially performed on sports, excite both players and audiences. Yet, boring games caused by huge differences of physical abilities between players never attract them. This research investigates how technology (artificially) dramatizes tight games, and empirically survey how both players enjoy the put-up games.

As references on our developments, realtime physically assisting technologies make ones succeed to play with physical motions $[1,2]$. While the physical assistance enables to fill the gap of ability between players, however, the obvious 'explicit' interventions squeeze senses of being in control-the sense of agency-out of the players. Without the sense of agency, players will be discouraged to engage with a game, since they obviously noticed that the game is put-up by something (one). Applying previous knowledge, we challenge to artificially make a physical tight game or sport played by multiple people, carefully managing players' sense of agencies.

We propose an implicit physical intervention system called The Tight Game for the 'tug of war' as an one-dimensional physical game. Our system includes four force sensors in the tug and two hidden high torque motors to provide realtime physical assistance. We designed the implicit assistance by leveraging human recognition of external force in physical actions. In a game, one pair of 


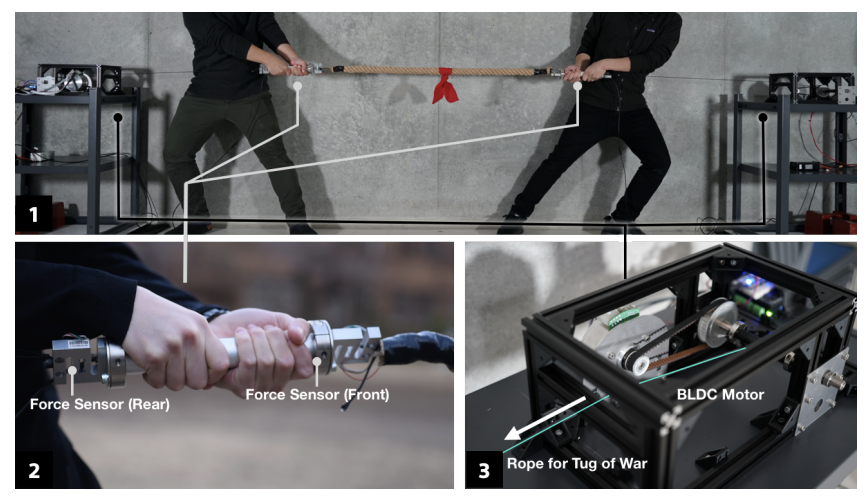

Figure 2: 1) The players are pulling the handles with the rope. 2) Two force sensors with the handle measure forces at both front and rear sides, which detects how each side of player(s) is putting force. 3) A BLDC motor is also pulling the rope.

players pulls the tug and believe that they are having a tight game. But, the system or even another person enables to mediate the game while the players will not recognize the physical interventions.

\section{THE TIGHT GAME SYSTEM}

A unit of our system consists of 1) two pairs of force sensors on the handle (Fig. 2-2) connected with the rope, and 2) a high torque motor (Fig. 2-3) at the end of the rope, which are implemented in two sides of players. We adopt a large-diameter brushless DC (BLDC) motor with a low reduction ratio, because of its backdrivability and the high torque output. Those are connected to a main computer which controls the torque outputs according to the force sensor signals and the assistance rate for each player, mentioned later. We set the two motor units hidden from the players not to be aware of the presence of the physical assistance.In addition to the hardware units, the remote user interface (Fig. 1-2) is also provided for a mediator who allows to control and intervene in the game without being noticed by the players.

\section{IMPLICIT FORCE ASSISTANCE DESIGN}

Providing interventions not noticed by the players, we designed the realtime force assistance by leveraging forces fitting to humans' recognition. Each motor output force $F m_{i}$ are described as;

$$
F m_{i}=R_{i} f\left(F s_{1}+F s_{2}\right)+F c(i=1,2)
$$

Here, $f\left(F s_{1}+F s_{2}\right)$ defines the reactive force assistance depending on the pulling force between both sides, which is a non-linear processing. This induces the sense of force assistance for the players, because the processing keeps force assistance in the range of force by humans based on the Weber-Fechner law in the output force and the perceived force in muscle activity (e.g., [3]) $F c$ is the constant force to prevent a slack of the rope.

With this reactive force assistance, the system or/and the mediator can control the balance of the force assistance between two players by changing $R_{1}$ and $R_{2}$ (Here, $R_{1}+R_{2}=1.0$ ). From our preliminary experiment, we found that naive (sudden) control of the assistance rate also resulted in clear discrimination of the existence

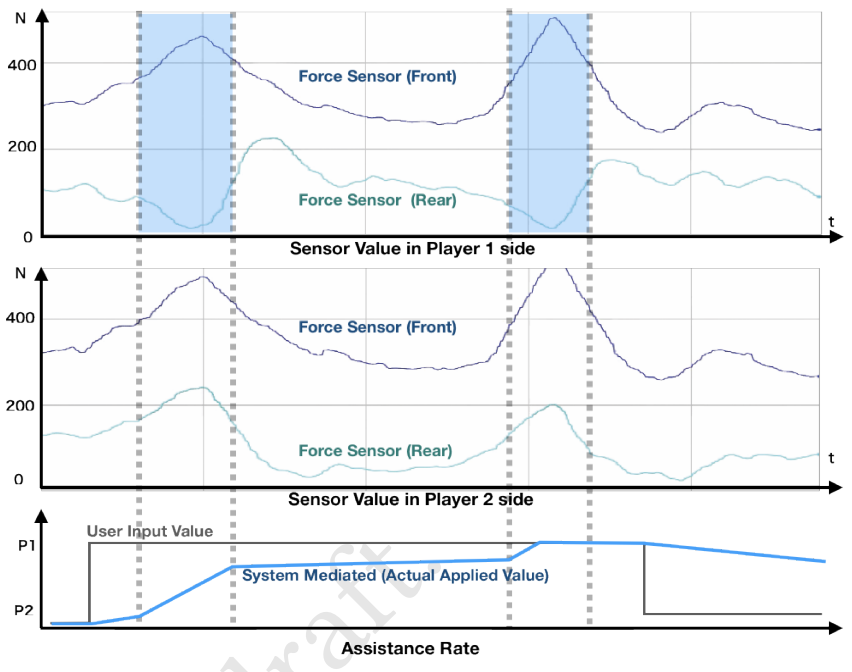

Figure 3: The system detects players' impulse pulling from the force sensors. Only when the player who will be assisted (Player1) gives a big pull, the assistance rate updates quickly.

of external force, since the weight of tug changes asynchronously with the players' actions. Therefore, we also set players' actions triggering to control the assistance rate. As depicted in Fig 3, the system detects the players' impulse pulling with the difference within the pair of force sensor values. The actually applying assistance rates $\left(R_{1}\right.$ and $\left.R_{2}\right)$ are gradually following the mediator's input. Only when the player who will be assisted gives a big pull, the assistance rate will quickly update according to the mediator's input.

\section{EXPERIENCE AT SIGGRAPH 2020}

A set of equipment is set at our booth. The attendees can choose to perform as a player (Fig. 1-1) or a mediator (Fig. 1-2, 3). The motor units (Fig. 2-3) will be hidden for the players who may not recognize if any intervention given or not. Even if just observing the game, the attendees can also enjoy seeing how a put-up tight game goes on. Our demonstration totally illustrates how the implicit force intervention works for a physical game: tug of war in this case.

\section{ACKNOWLEDGMENTS}

This work is supported by JST ERATO Grant Number JPMJER1701, Japan.

\section{REFERENCES}

[1] Shunichi Kasahara, Jun Nishida, and Pedro Lopes. 2019. Preemptive Action: Accelerating Human Reaction Using Electrical Muscle Stimulation Without Compromising Agency. In Proceedings of the 2019 CHI Conference on Human Factors in Computing Systems (Glasgow, Scotland Uk) (CHI '19). ACM, New York, NY, USA, Article 643, 15 pages. https://doi.org/10.1145/3290605.3300873

[2] Azumi Maekawa, Seito Matsubara, Atsushi Hiyama, and Masahiko Inami. 2019. PickHits: Hitting Experience Generation with Throwing Motion via a Handheld Mechanical Device. In ACM SIGGRAPH 2019 Emerging Technologies (Los Angeles, California) (SIGGRAPH '19). Association for Computing Machinery, New York, NY, USA, Article Article 20, 2 pages. https://doi.org/10.1145/3305367.3327996

[3] Kazuhiro Takemura, Naoki Yamada, Atsuhide Kishi, Kazuo Nishikawa, Takahide Nouzawa, Chang Li, Yuichi Kurita, and Toshio Tsuji. 2013. A Subjective Force Perception Model of Humans and Its Application to a Steering Operation System of a Vehicle. In 2013 IEEE International Conference on Systems, Man, and Cybernetics. IEEE, Manchester, 3675-3680. https://doi.org/10.1109/SMC.2013.626 